\title{
Biomarkers in colorectal cancer: the future is getting closer
}

\author{
Desamparados Roda · Andrés Cervantes
}

$\mathrm{C}$ olorectal cancer is the third most common cancer worldwide and the second leading cause of cancerrelated death in the Western world. Approximately $75 \%$ of patients with colorectal cancer present with localised disease, whereas metastatic disease accounts for $25 \%$ of newly diagnosed patients. Systemic treatment is based on cytotoxic agents and monoclonal antibodies targeting epidermal growth factor receptor (EGFR) and vascular endothelial growth factor (VEGF). In the last decade, the optimal use of these agents in patients with metastatic disease has dramatically improved median survival from 6 to 24 months. However, despite all these therapeutic advances, the prognosis of patients with metastatic colorectal cancer remains poor, and, apart from those having surgery of isolated metastases, long-term survival is very low.

In the adjuvant setting, 5-fluorouracil- and oxaliplatinbased chemotherapy has demonstrated a significant improvement in disease-free and overall survival, particularly in patients with resected stage III disease. The quantitative benefit of adjuvant therapy in patients with stage II colorectal cancer is less important and remains a matter of controversy. However, despite this strategy, almost $40 \%$ of patients diagnosed initially with localised disease will develop recurrences. Therefore, there is a need to better identify these patients at risk and to adapt to them what we could consider optimal treatment. Anatomic criteria defined by Dukes [1] almost 80 years ago are currently the basis of the tumour/node/metastasis (TNM) staging system upon which most clinical decisions concerning postoperative treatment are based.

\footnotetext{
D. Roda $\cdot$ A. Cervantes

INCLIVA

University of Valencia

Valencia, Spain

D. Roda $\cdot$ A. Cervantes $(\bowtie)$

Hematology and Medical Oncology Department

Hospital Clínico Universitario de Valencia

Avda. Blasco Ibáñez, 17

ES-46010 Valencia, Spain

e-mail: andres.cervantes@uv.es
}

However, some changes are expected to occur in the molecular medicine era. A high number of articles are reporting on the importance of several molecular markers, either predictive or prognostic, to help clinicians make better decisions with cancer patients. In light of this challenge, researchers are attempting to determine a more rational and pathogenic approach to cancer therapy. Those findings will eventually give rise to personalised medicine, following the wise, classical, Hypocratic aphorism: "There is no disease, but sick people".

In this issue of Clinical and Translational Oncology, Markman et al. [2] provide a well-written and comprehensive review aiming to highlight the prognostic and predictive biomarkers in colorectal cancer. An extensive amount of information is being published on many different mechanisms that may have therapeutic interest in colorectal cancer, but the important point is how to apply them properly in clinical practice. Improved understanding of cancer biology and advances in biotechnology bring us closer to the concept of individualised treatment. A key component of this new paradigm is development of biomarkers that can guide application of new or even existing treatments. This requires a deep understanding of the relationship between biomarker and treatment effect.

Randomized phase III trials are designed to estimate clinical benefit by adding an experimental drug to standard treatment. Some recently published randomised studies are also designed to assess the importance of specific biomarkers. In others, the authors attempt to justify a retrospective assessment of certain biomarkers. Several experts have emphasised the importance of doing such an assessment with some important methodological requirements [3, 4]. When a new drug is developed, we all know that a complex methodological path has to be completed. In general, we need phase I data indicating the maximum tolerated dose and defining dose-limiting toxicities as well as pharmacokinetic and pharmacodynamic end points. Antitumor activity should be well established in phase II studies thereafter, and eventually, phase III data will determine the benefit over standard therapy. None of us will be using a new agent, apart from those included in well-designed clinical studies, without that agent being approved by the corresponding drug agency. Similarly, a newly developed biomarker should be adequately tested over several phases, 
and at the very end, they should be validated in randomized trials.

When the predictive role of v-Ki-ras2 Kirsten rat sarcoma viral oncogene homolog (KRAS) was explored in patients with advanced colon cancer receiving antiEGFR drugs, consistent results from clinical trials firmly concluded that KRAS mutation is a negative predictor of response to anti-EGFR therapy. Nowadays, KRAS status testing is required prior to initiation of anti-EGFR antibodies. Due to the complexity of the EGFR signalling system, it is likely that predictive algorithms will be developed for metastatic colorectal cancer, thus adding other molecular biomarkers. For instance, combining KRAS mutational status with the presence of b-Raf proto-oncogene serine/ threonine-protein kinase $(B R A F)$ and/or phosphoinositide3-kinase, catalytic, alpha polypeptide (PIK3CA) mutations and phosphatase and tensin homolog (PTEN) loss of function might identify additional patients who are unlikely to respond to treatment with EGFR-targeted monoclonal antibodies. However, these additional markers require further validation before they can be incorporated into clinical practice. On the other hand, all responding tumours eventually escape from EGFR-targeted treatment (i.e. develop acquired resistance). Even the best responses obtained in KRAS/BRAF wild-type tumours are transient, and they do not last longer than 12-18 months. Therefore, another urgent question is to identify the mechanisms of secondary resistance to anti-EGFR therapies in colorectal cancer patients $[5,6]$.

Further work is also required to explore potential early markers of response that can be incorporated into the design of future prospective clinical trials and guide therapeutic decisions regarding continuation of treatment in individual patients. In 1990, Fearon and Vogelstein presented their well-known model for the genetic basis of colorectal neoplasia. They proposed that "identification of the genetic alterations present in tumours may provide a molecular tool for improved estimation of prognosis in patients with colorectal cancer" [7]. Nowadays, almost two decades later, we still have much to learn about the prognostic or predictive value of these and others "colorectal cancer biomarkers".

Molecular profiling of tumours may certainly let us identify patients who are more likely to benefit from a specific therapy. This would stimulate clinicians to tailor treatment according to an individual patient and/or tumour profile, converting the so-called personalised or tailored therapy into a reality. New methodologies such as microarray-based gene expression profiling, proteomic profiling, comparative genomic hybridisation analysis and metabolomics would allow tumour samples to be profiled on a global scale $[8,9]$. The most frequently used genomewide approach is DNA microarray profiling. One platform is the Oncotype DX colon cancer test being used to profile gene expression. Molecular alterations are used to redesign the taxonomy of solid tumours by moving it from a histologically to a genetically based level.

Parallel development of predictive molecular and clinical markers is paramount to achieve the best outcomes from targeted treatments, and KRAS is so far the only validated predictive molecular marker in colorectal cancer for EGFR-directed monoclonal antibodies. In the future, we expect to employ a personalised or tailored therapy that will become a reality, with less toxicity and superior efficacy. This will become a reality only if we are able to improve our molecular knowledge, we can consistently validate these new biomarkers and we can eventually apply them in our clinical decisions. Clinical and translational research that is in progress will hopefully help to provide the much promised hope of personalised medicine in the management of colorectal cancer. We can foresee that in some years, the treatment for advanced colon cancer will be based upon molecular features. The future is not yet here, but we are getting closer.

\section{References}

1. Dukes C (1937) Histological Grading of Rectal Cancer: (Section of Pathology) Proc R Soc Med 30:371-376

2. Markman B, Rodríguez-Freixinos V, Tabernero J (2010) Biomarkers in colorectal cancer Clin Transl Oncol 12:261-270

3. Mandrekar SJ, Sargent DJ (2009) Clinical trial designs for predictive biomarker validation: theo- retical considerations and practical challenges. J Clin Oncol 27:4027-4034

4. Freidlin B, MacShane MN, Korn EL (2010) Randomized clinical trials with biomarkers: design issues. J Natl Cancer Inst 102:152-160

5. Siena S, Sartore-Bianchi A, Di Nicolantonio F et al (2009) Biomarkers predicting clinical outcome of epidermal growth factor receptor-targeted therapy in metastatic colorectal cancer. J Natl Cancer Inst 101:1308-1324

6. Bardelli A, Siena S (2010) Molecular mecha- nisms of resistance to cetuximab and panitumum$\mathrm{ab}$ in colorectal cancer. J Clin Oncol 28(7):12541261

7. Fearon ER, Vogelstein B (1990) A genetic model for colorectal tumorigenesis. Cell 61:759-767

8. Walther A, Johnston E, Swanton C et al (2009) Genetic prognosis and predictive markers in colorectal cancer. Nat Rev Cancer 9:489-499

9. Sjoblom T, Jones S, Wood LD et al (2006) The consensus coding sequences of human breast and colorectal cancers. Science 314:268-274 